\title{
Vortex-flow electromagnetic emission in stacked intrinsic Josephson junctions
}

\author{
Myung-Ho Bae and Hu-Jong Lee \\ Department of Physics, Pohang University of Science and Technology, Pohang 790-784, Republic of Korea
}

(Dated: June 28, 2018)

\begin{abstract}
We confirmed the existence of the collective transverse plasma modes excited by the motion of the Josephson vortex lattice in stacked intrinsic Josephson junctions of $\mathrm{Bi}_{2} \mathrm{Sr}_{2} \mathrm{CaCu}_{2} \mathrm{O}_{8+x}$ by observing the multiple subbranches in the Josephson-vortex-flow current-voltage characteristics. We also observed the symptom of the microwave emission from the resonance between the Josephson vortex lattice and the collective transverse plasma modes, which provides the possibility of developing Josephson-vortex-flow electromagnetic oscillators.
\end{abstract}

PACS numbers: 74.72.Hs, 74.50.+r, 74.78.Fk, 85.25.Cp

The generation of THz-range electromagnetic waves using the Josephson-vortex dynamics in naturally stacked $\mathrm{Bi}_{2} \mathrm{Sr}_{2} \mathrm{CaCu}_{2} \mathrm{O}_{8+x}$ (Bi-2212) intrinsic Josephson junctions (IJJs) has been attempted extensively because of the coherent (thus, high-power), continuous, and frequency-tunable characters of the generated waves 1]. The characters of this technique are distinctive from those by other attempts [2] based on quantum cascade, relativistic electron bunches, and optical parametric control. This electromagnetic emission from the Josephson vortex system is induced by the resonance between moving Josephson vortices and the collective transverse plasma (CTP) modes in the stacked IJJs 3 .

A stack of Bi-2212 containing $N$ IJJs exhibits $N$ eigen CTP modes, which can be excited by the moving Josephson vortex lattice (JVL) that forms in a high magnetic field [4]. If the frequency of the temporal oscillation of the phase difference across a junction due to moving JVL matches with that of a transverse plasma mode, a resonant plasma oscillation is excited with microwave emission at the boundary of stacked IJJs. The JVL also transforms its lattice configuration along the $c$-axis direction in accordance with the $c$-axis standing-wave mode of the strongly amplified plasma oscillations. The resonance of JVL to the CTP modes appears as the multiple collective Josephson vortex-flow branches in the tunneling currentvoltage $(I-V)$ curves of stacked junctions [5].

We report the observation of the CTP modes induced by the JVL motion and the excitation of corresponding electromagnetic waves in a stack of IJJs in Bi-2212. The existence of the CTP modes was confirmed by observing the multiple branches in the Josephson vortex-flow region. In addition, for a proper bias, the emission of the electromagnetic waves by the collective vortex resonance motion in a stack of IJJs (the oscillator stack) was examined using another stack of IJJs (the detector stack), which was placed within a fraction of $\mu \mathrm{m}$ from the oscillator stack. The microwave emission from the oscillator stack and the resulting irradiation onto the detector stack was evidenced by the suppression of the tunneling critical current revealed in the quasiparticle branches and the increase of Josephson vortex-flow voltages in the detector stack. Our numerical calculation for the effect of microwave irradiation on the detector stack was also

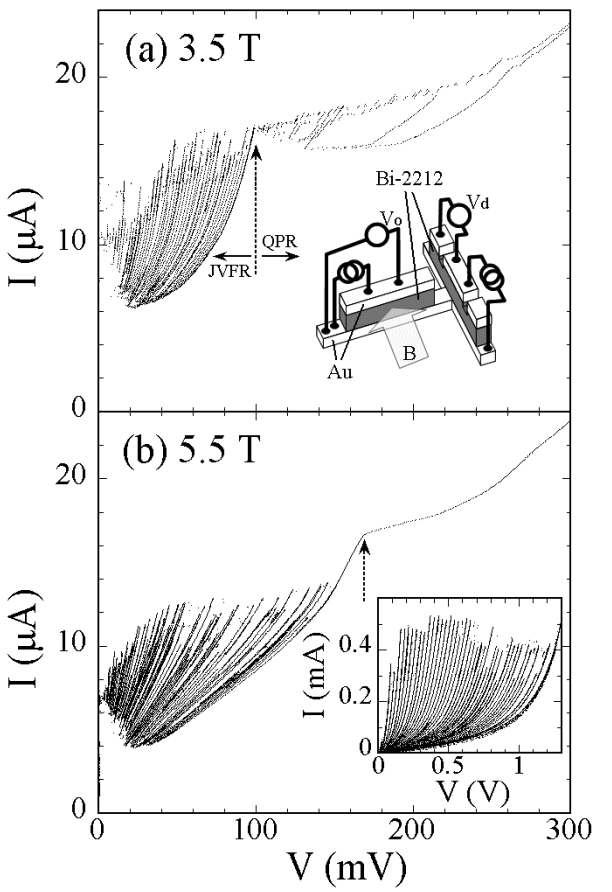

FIG. 1: The $H$-field dependence of the Josephson-vortex-flow branches and the quasiparticle branches of SP1 in (a) $H=3.5$ $\mathrm{T}$ and (b) $5.5 \mathrm{~T}$. Inset of (a): the sample configuration. Inset of (b): multiple quasiparticle branches of SP1 in zero field.

consistent with our observed results.

Bi-2212 single crystals, prepared by the conventional solid-state-reaction method, were slightly overdoped. We fabricated, using the double-side cleaving technique 6], two samples of IJJs, each sandwiched between two Au electrodes at its top and bottom without the basal part [the inset of Fig. 1(a)]. Adopting the geometry with the basal part eliminated enabled us to measure the Josephson vortex dynamics in coupled IJJs without the interference of the vortex motion in the basal stack. The detailed fabrication procedure is described in Ref. 7]. In the inset of Fig. 1(a) the left and right stacks are the oscillator and detector stacks, respectively. The lateral size of each oscillator of two samples was $16 \times 1.5 \mu \mathrm{m}^{2}$ [SP1] and 15 
$\times 1.4 \mu \mathrm{m}^{2}$ [SP2], respectively. The magnetic field was aligned in parallel with the junction planes within 0.01 degree to avoid the pinning of Josephson vortices by the formation of pancake vortices in $\mathrm{CuO}_{2}$ bilayers.

Figs. 1(a) and (b) show the magnetic field dependence of $I$ - $V$ curves of SP1 at $4.2 \mathrm{~K}$ in 3.5 and $5.5 \mathrm{~T}$, respectively. The contact resistance caused by the two-terminal configuration adopted was subtracted numerically. The current-bias point corresponding to the dotted vertical arrow in each figure originates from the zero-field Josephson critical current. Thus, the voltage-bias region below the critical point corresponds to the pair-tunneling state, although it is resistive due to the Josephson vortex flow in high fields. The set of multiple branches in the voltage (McCumber) bias region above the critical point are the quasiparticle branches, while those below the critical point are the collective Josephson vortex-flow branches [5]. Estimated from the number of zero-field quasiparticle branches as in the inset of Fig. 1(b) for SP1, stacks in SP1 and SP2 contained 45 and 22 IJJs, respectively.

The numbers of the Josephson-vortex-flow branches below the critical bias points for SP1 and SP2 are $\sim 42$ and $\sim 18$ (not shown), respectively, which are similar to the numbers of the IJJs in the respective samples. In addition, the multiple Josephson-vortex-flow branches become clearer and wider for a higher transverse magnetic field, which is in contrast to the shrinking quasiparticle branches with increasing fields [see Fig. 1(a) and (b)].

The collective transverse plasma oscillation excited by the moving JVL is expected to emit electromagnetic waves at the junction edge [8]. Detection of this microwave emission, in turn, would be more positive confirmation that the observed multiple branches resulted from the moving JVL in resonance with the CTPs. A separate stack for the microwave detection was positioned a fraction of $\mu \mathrm{m}$ apart from the oscillator stack. In the direction facing the applied field, the detector was 0.7 $\mu \mathrm{m}$ wide, which was longer than the Josephson penetration depth of $0.3 \mu \mathrm{m}$ and thus was in a long-junction limit. The oscillator and the detector stacks, connected by the bottom Au common-ground electrode, acted as a microwave coupler [see the inset of Fig. 1(a)]. The frequency of emitted microwaves may reach a $\mathrm{THz}$ range so that a conventional Nb-based Josephson detector cannot be used, because its gap size is smaller than the energy of the emitted waves.

The response of IJJs in the detector stack to the emitted microwaves from the oscillator stack may be revealed as the appearance of the Shapiro steps, the suppression of the $c$-axis tunneling critical current, $I_{c}$, revealed in the quasiparticle branches, or the increase of the voltage of the low-bias region due to the resistive motion of microwave-induced vortices [9, 10]. The inset of Fig. 2 shows the bias conditions, $V_{o s c}$, displayed by dots in the Josephson vortex-flow branches of the oscillator stack of $\mathrm{SP} 1$ in $3 \mathrm{~T}$. The black $I-V$ curves in the main panels are the quasiparticle branches of the detector stack without any bias in the oscillator stack. For the biases of

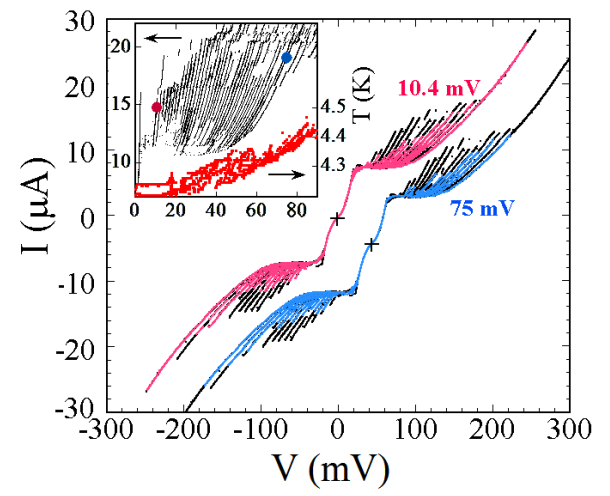

FIG. 2: (Color online) Response of the quasiparticle branches of the detector stack of SP1 to biasing the oscillator stack. The curves are shifted for clarity. Inset: the bias condition of the oscillator stack as denoted by dots and the corresponding local temperature of the detector stack, with the base temperature at $4.2 \mathrm{~K}$, for varying bias in the oscillator stack.

$V_{\text {osc }}=10.4$ and $75 \mathrm{mV}$ (corresponding to 110 and $800 \mathrm{GHz}$ [1] , respectively) in the oscillator, $I_{c}$ in the quasiparticle branches of the detector stack is suppressed significantly. Our previous studies 7, 10 confirmed that the irradiation of microwaves on the IJJs suppresses the $I_{c}$ revealed in the quasiparticle branches.

The temperature increase of the detector stack by the self-heating in the oscillator for a finite dc bias may cause the similar suppression of the $I_{c}[12$. We directly monitored the actual temperature variation in the detector for any finite bias in the oscillator. Details of the thermometry are explained in Ref. 13], which confirms that both stacks in our measurement configuration should be at an identical temperature. The inset of Fig. 2 shows that the actual temperature increase in this measurement turned out to be less than $4.5 \mathrm{~K}$, so that the observed suppression of the $I_{c}$ around $4.2 \mathrm{~K}$ should not have been caused by the bias-induced self-heating. One may also attribute the behavior to the leakage of the bias current from the oscillator to the detector through the bottom Au commonground electrode, rather than the microwave emission. However, any shift of the $I-V$ curves due to a dc offset current in the detector was not observed for any biases used. We thus attribute the suppression of the $I_{c}$ in the quasiparticle branches to the response of the stacked IJJs in the detector to the mircowave irradiation.

Fig. 3 shows the change in the Josephson vortex-flow branch in the detector of the specimen SP2 with varying the bias voltage $V_{o s c}$ of its oscillator. For $V_{o s c}=4.5$ $\mathrm{mV}$ (corresponding to $100 \mathrm{GHz}$ ) little variation of the $I$ - $V$ curves from the zero-bias condition is visible. The bias of $4.5 \mathrm{mV}$ corresponds to the near-triangular JVL in the oscillator stack, which represents the out-of-phase Josephson vortex distribution between adjacent junctions and thus corresponds to the weakest emission power, as illustrated in Figure 3. For $V_{\text {osc }}=18.1 \mathrm{mV}$ (corresponding to $400 \mathrm{GHz}$ ), however, a distinct increase of the Joseph- 


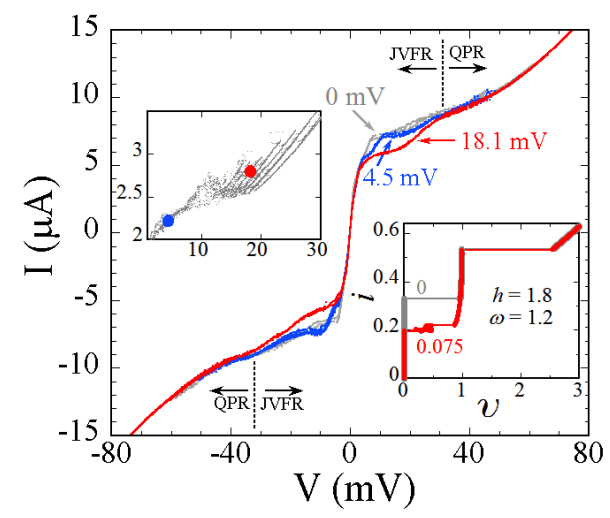

FIG. 3: (Color online) The change in the Josephson vortexflow branch in the detector of SP2 for the bias voltage of its oscillator $V_{o s c}=0,4.5$, and $18.1 \mathrm{mV}$. Upper inset: bias condition (denoted by dots) of the oscillator stack. Lower inset: numerically calculated $I-V$ curves for the ac $(\omega=1.2)$ field component of $h_{a c}=0$ and 0.075 in a $d c$ magnetic field of $h=1.8$.

son vortex-flow voltage from the zero-bias condition is seen. We interpret it as arising from the flow of additional Josephson vortices generated by the magneticfield component of emitted higher-intensity microwaves from the oscillator with JVL close to the highly coherent square-lattice configuration.

We numerically calculated vortex-flow feature, in the detector stack induced, by the magnetic-field component of any irradiated microwaves [14]. For simplicity, we consider a detector consisting of a single Josephson junction. The perturbed sine-Gordon equation for the dynamics of the phase difference across a Josephson junction, $\phi$, is

$$
\frac{\partial^{2} \phi}{\partial x^{2}}-\frac{\partial^{2} \phi}{\partial t^{2}}-\sin \phi=\alpha \frac{\partial \phi}{\partial t}-i-i_{a c} \sin \omega t
$$

where the spatial and time variables of the $\phi$ are normalized by the Josephson penetration depth $\lambda_{J}$ and by the inverse of the Josephson plasma frequency, $\omega_{p}^{-1}$, respectively. The coefficient $\alpha$ is the damping parameter due to the quasiparticle tunneling, $\omega$ is the ac frequency normalized by $\omega_{p}^{-1}$ and $i\left(i_{a c}\right)$ is the $d c(a c)$ bias current normalized by the tunneling critical current $I_{c}$. We adopted the boundary condition in the external $d c$ and $a c$ magnetic fields as $\partial \phi / \partial x(x=0, t)=h-h_{a c} \sin \omega t$ and $\partial \phi / \partial x(x=l, t)=h$, where $l$ is the junction length normalized by $\lambda_{J}, h \equiv 2 \pi N_{f} / l$, and $N_{f}$ is the number of the Josephson vortices. The averaged voltage, $v$, in the $x$ axis is normalized as $\langle\partial \phi / \partial t\rangle l / 2 \pi$.

The lower inset of Fig. 3 shows the simulation results for a $d c$ magnetic field of $h=1.8$ without and with the irradiation of the external electromagnetic waves, where we used parameter values of $l=3, \omega=1.2$, and $\alpha=0.1$. The simulation for the microwave irradiation mimics the observation in the detector as in Fig. 3: the appearance of the finite Josephson-vortex-flow voltage for $0.2<i<0.32$ in the inset. The vertical step at $v=1$ is the resonant Josephson-vortex-flow branch. The numerical results suggest that the Josephson vortices induced by the $a c$ magnetic-field component of the electromagnetic waves effectively play the role of a $d c$ magnetic field of the corresponding magnitude, which is qualitatively consistent with our experimental observation in Fig. 3 and previous reports [15].

The microwave emission from the resonance between the Josephson vortex motion and the collective plasma oscillation modes, as manifested by changing $I$ - $V$ curves in the detector stack, provides a possibility of application to the Josephson vortex-flow oscillator. The observation of the Shapiro steps in the detector stack would be the most convincing evidence for the microwave generation from the oscillator stack. Recently it was theoretically predicted [1] that the radiation power from $\mathrm{Bi}$ 2212 Josephson-vortex-flow oscillator in an optimal condition can be as high as $\sim 2000 \mathrm{~W} / \mathrm{cm}^{2}$ from the edge of the stack, which corresponds to about $1 \sim 2 \mu \mathrm{W}$ in our samples. Observing Shapiro steps requires sufficiently strong emission power with improved matching between the oscillator and detector stacks. Thus, devising optimal impedance matching schemes, using antennas or dielectric wave guides [1], is the most essential step for the decisive confirmation of the microwave emission as well as the practical application of the Josephson vortex-flow motion to the electromagnetic local oscillator.

This work was supported by the National Research Laboratory program administrated by Korea Science and Engineering Foundation and also by the AFOSR/AOARD of the US Air Force under Contract No. FA5209-04-P-0253.
[1] M. Tachiki, M. Iizuka, K. Minami, S. Tejima, and H. Nakamura, Phys. Rev. B 71, 134515 (2005).

[2] R. Köhler, A. Tredicucci, F. Beltram, H. E. Beere, E. H. Linfield, A. G. Davies, D. A. Ritchie, R. C. Iotti, and F. Rossi, Nature 417, 156 (2002); G. L. Carr, M. C. Martin, W. R. McKinney, K. Jordan, G. R. Neil, and G. P. Williams, Nature 420, 153 (2002); K. Kawase, H. Minamide, K. Imai, J.-I. Shikata, and H. Ito, Appl. Phys. Lett. 80, 195 (2002); W. Shi and Y. J. Ding, Appl. Phys.
Lett. 84, 1635 (2004).

[3] R. Kleiner, Phys. Rev. B. 50, 6919 (1994); M. Machida, T. Koyama, A. Tanaka, and M. Tachiki, Physica C 330, 85 (2000).

[4] M. Machida and M. Tachiki, Curr. Appl. Phys. 1, 341 (2001).

[5] M.-H. Bae and H.-J. Lee, Phys. Rev. B 70, 052506 (2004).

[6] H. B. Wang, P. H. Wu, and T. Yamashita, Appl. Phys. 
Lett. 78, 4010 (2001).

[7] M.-H. Bae, H.-J. Lee, J. Kim, and K.-T. Kim, Appl. Phys. Lett. 83, 2187 (2003).

[8] G. Hechtfischer, R. Kleiner, A. V. Ustinov, and P. Müller, Phys. Rev. Lett. 79, 1365 (1997).

[9] Y.-J. Doh,J. Kim, K.-T. Kim, and H.-J. Lee, Phys. Rev. B. 61, R3834 (2000); H. B. Wang, P. H. Wu, and T. Yamashita, Phys. Rev. Lett. 87, 107002 (2001); Yu. I. Latyshev, M. B. Gaifullin, T. Yamashita, M. Machida, and Y. Matsuda, Phys. Rev. Lett. 87, 247007 (2001).

[10] Y.-J. Doh, J. Kim, H.-S. Chang, S. Chang, H.-J. Lee, K.-T. Kim, W. Lee, and J.-H. Choy, Phys. Rev. B. 63,
144523 (2001).

[11] The frequency is estimated based on the frequencyvoltage (per junction) conversion relation, $f=2 e V_{o s c} / N h$ $\left(=483.6 \mathrm{GHz} / \mathrm{mV} \times V_{o s c} / N\right)$.

[12] Private communications with E. Kume.

[13] M.-H. Bae, J.-H. Choi, and H.-J. Lee, Appl. Phys. Lett. 86, 232502 (2005).

[14] E. Goldobin (2003), URL http://www.geocities.com/SiliconValley/Heights/7318/StkJJ.htm.

[15] F. L. Barkov, M. V. Fistul, and A. V. Ustinov, Phys. Rev. B. 70, 134515 (2004). 